\title{
CALIDAD DE VIDA LABORAL EN LAS PYMES DE BRASIL: UN DIAGNÓSTICO
}

\section{QUALITY OF LIFE IN BRAZILIAN S\&ME'S: A DIAGNOSTIC STUDY}

\author{
Emigdio Larios Gómez*, Rosa María Mora Morales**, \\ Ana Hermelinda Vargas Carrillo ${ }^{* \star}$, Samanta de Salazar Calvo****
}

* Doctor en Ciencias Administrativas por el IPN Esca-Santo Tomás. Profesor Investigador de Posgrado de la Universidad de Oriente. ORCID: http://orcid.org/0000-0002-3514-1319

** Maestra en la Benemérita Universidad Autónoma de Puebla, Campus Tehuacán. Email: rosamaria_rosy@hotmail.com. ORCID: http://orcid.org/0000-0002-4185-8818

*** Maestra en la Benemérita Universidad Autónoma de Puebla, Campus Tehuacán. ORCID: http://orcid.org/0000-0001-5440-1094

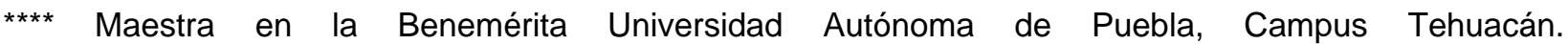
ORCID: http://orcid.org/0000-0002-5012-9019

Dirección para recibir correspondencia: herr.larios@gmail.com 
CALIDAD DE VIDA LABORAL EN LAS PYMES DE BRASIL: UN DIAGNÓSTICO

RESUMEN

OBJETIVO: Analizar el nivel de calidad de vida laboral en Pequeñas y Medianas Empresas (MyPEs) de Brasil, que permita identificar puntos clave para la gestión eficiente del capital humano.

MATERIAL Y MÉTODO: Se desarrolló una investigación empírica con un enfoque cuantitativo de tipo transversal, no experimental, se aplicó una encuesta a empresas MyPEs en ciudad de Piracicaba en el Estado de São Paulo, Brasil. La muestra fue por conveniencia de 14 empresas, clasificadas por el Servicio Brasileño de Apoyo a las Micro y Pequeñas Empresas (SEBRAE) como MyPE. Fueron cuatro microempresas del sector comercial y 10 pequeñas empresas del sector servicios.

RESULTADOS: Se presentan de forma global (promedio) la estadística aplicada a las empresas comerciales y de servicios, debido a que los resultados individuales por sector variaron en menos del 5\%, entre las medias y la desviación estándar de cada sector.

CONCLUSIONES: Un empleado satisfecho personalmente, rendirá más en la empresa, será más productivo y mayor lealtad también. Asimismo, esto influye en el nivel de vida laboral que tengan los empleados; es decir, un empleado con altos niveles de calidad de vida en su familia, en el mismo y con la empresa o puesto que tenga, repercutirá en la satisfacción integral en la empresa.

PALABRAS CLAVE: Satisfacción laboral. Clima laboral. Competitividad laboral. PyMEs. 
CALIDAD DE VIDA LABORAL EN LAS PYMES DE BRASIL: UN DIAGNÓSTICO

\section{ABSTRACT}

OBJECTIVE: To analyze the level on quality in the working environment in Small \& Medium Enterprises in Brazil, that allows to identify key points for an efficient workforce management.

MATERIAL AND MHETOD: An empirical non-experimental research was carried out. A questionnaire was administered in S\&ME's in Piracicaba city in São Paulo, Brazil, to a convenient sample of 14 enterprises, classified by Servicio Brasileño de Apoyo a las Micro y Pequeñas Empresas (SEBRAE) or MyPE. 4 microenterprises from the commercial sector and 10 from the service sector.

RESULTS: Statistics as presented in a global format, since individual results per sector showed a variance of less than $5 \%$, in the mean and standard deviation in each sector.

CONCLUSIONS: A satisfied employee will, at a personal level, have a better performance, be a more productive and more loyal employee. Thus influencing the working environment, an employee with high quality of life, both family and laboral lives, this aspect will have a positive impact in the integral satisfaction towards the company.

KEY WORDS: Laboral Satisfaction. Working environment. Laboral Competitiveness. S\&ME's.

\section{INTRODUCCIÓN}

Martínez, Sánchez, Santero y Marcos (2009) sugieren que los indicadores clave que inciden en la competitividad son los indicadores externos, que tienen que ver con el entorno de los negocios, la tecnología e innovación, y se mide en la capacidad para lanzar nuevos productos al mercado, la calidad, la gestión de los recursos humanos, las capacidades directivas en el uso de nuevas herramientas de gestión, la internacionalización entendida como la capacidad para llegar a los mercados externos y el financiamiento como una fuente necesaria para su permanencia. Con base en las aportaciones de los autores mencionados (ver tabla 1), los indicadores propuestos, que se han tomado en cuenta para medir la competitividad de la empresa. 
CALIDAD DE VIDA LABORAL EN LAS PYMES DE BRASIL: UN DIAGNÓSTICO

Tabla 1

Indicadores de competitividad empresarial

\begin{tabular}{|c|c|c|c|c|c|c|}
\hline Indicador/Autor & $\begin{array}{c}\text { Rubio y } \\
\text { Aragón } \\
\text { (2006) }\end{array}$ & $\begin{array}{c}\text { De la Cruz } \\
\text { Morales, y } \\
\text { Carrasco } \\
(2006)\end{array}$ & $\begin{array}{c}\text { Solleiro y } \\
\text { Castañón } \\
(2005)\end{array}$ & $\begin{array}{l}\text { OCDE } \\
(1992)\end{array}$ & $\begin{array}{c}\text { Quiroga } \\
\text { (2003) }\end{array}$ & $\begin{array}{c}\text { Martínez, } \\
\text { Santero, } \\
\text { Sánchez y } \\
\text { Marcos (2009) }\end{array}$ \\
\hline $\begin{array}{l}\text { Indicadores } \\
\text { Externos }\end{array}$ & & & $x$ & & $x$ & $x$ \\
\hline Tecnología & $x$ & $x$ & $x$ & & $x$ & $x$ \\
\hline Innovación & $x$ & & & & & $x$ \\
\hline Mercadotecnia & $x$ & $x$ & $x$ & $x$ & & \\
\hline Recursos Humanos & $x$ & $x$ & $x$ & $x$ & $x$ & $x$ \\
\hline $\begin{array}{l}\text { Capacidades } \\
\text { Directivas }\end{array}$ & $x$ & & & & $x$ & $x$ \\
\hline $\begin{array}{l}\text { Recursos } \\
\text { Financieros }\end{array}$ & $x$ & $x$ & $x$ & & $x$ & \\
\hline Cultura & $x$ & & & & & \\
\hline Calidad & $x$ & & $x$ & & $x$ & $x$ \\
\hline Producción & & $x$ & & $x$ & $x$ & \\
\hline Logística & & $x$ & & & & \\
\hline $\begin{array}{l}\text { Organización } \\
\text { Interna }\end{array}$ & & & $x$ & & $x$ & \\
\hline Compras & & & $x$ & $x$ & $x$ & \\
\hline $\begin{array}{l}\text { Investigación y } \\
\text { Desarrollo }\end{array}$ & & & $x$ & $x$ & $x$ & $x$ \\
\hline $\begin{array}{l}\text { Interacción con } \\
\text { Proveedores y } \\
\text { clientes }\end{array}$ & & & & $x$ & & \\
\hline Internacionalización & & & & & & $x$ \\
\hline Financiamiento & & & & & & $x$ \\
\hline
\end{tabular}

Fuente: Saavedra, Milla y Tapia (2013).

Desde este enfoque de la competitividad, en la actualidad pocas organizaciones se dan a la tarea de estudiar y analizar el clima laboral que hay entre los empleados, además de conocer lo que los empleados piensan de la empresa; sin embargo, el correcto análisis de la percepción de 


\section{CALIDAD DE VIDA LABORAL EN LAS PYMES DE BRASIL: UN DIAGNÓSTICO}

los trabajadores con respecto a las condiciones laborales, ergonómicas, ambientales, económicas, de seguridad y ascenso laboral es de vital interés, puesto de dicho análisis se pueden obtener grandes ideas y lograr mejoras para potencializar el capital intelectual con el que ya se cuenta. En algunas empresas, muchas veces se aplican las estrategias "tradicionales" para motivar al personal, en esta visión antigua se tiene como principal aspecto el que la gente se mueve por miedo, no quieren perder su trabajo y por lo tanto, hacen lo que les corresponde, sin embargo hay que buscar la mejor manera de obtener todo el rendimiento posible de los empleados y no basta con atemorizarlos para obtener productividad, puesto que la creatividad e innovación, no se da en ambientes hostiles en donde no hay cabida a la libertad (García y Larios, 2018).

Las organizaciones, sin importar el tipo de estas, se encuentran formadas por personas y las relaciones interpersonales se dan con el fin de desempeñar acciones que ayuden al logro de las metas. Tratar de entender el impacto que los individuos, los grupos y la estructura tienen sobre el comportamiento dentro de la organización, permite mejorar la eficacia de ésta y el alcance de sus objetivos, este comportamiento se da debido a la cultura organizacional que en ellas prevalece (Zamora, 2005). Reflejo de ello es el clima organizacional, por lo que para conocer las percepciones que el trabajador tiene de las características de la organización, que influyen en las actitudes y comportamiento de los empleados, es necesario elaborar diagnósticos de Clima Organizacional (CO). El desarrollo organizacional, surge en 1962 introducido por primera vez al área de psicología organizacional por Gellerman, como un conjunto de ideas sobre el hombre, la organización y el ambiente, con el propósito de facilitar el crecimiento y desarrollo de las organizaciones.

El concepto de calidad de vida laboral de Chiavenato (2011), que implica una serie de factores por medio de los cuales las personas satisfacen sus necesidades a través del trabajo; se relaciona con diversos factores, tales como: la satisfacción laboral, la motivación, las posibilidades de crecimiento en el trabajo, reconocimiento, salario, prestaciones, relaciones con los compañeros y jefes, libertad para decidir, participación en las decisiones que afecten su trabajo, así como ambiente físico y psicológico adecuados para el desarrollo de la labor. Con base en el análisis de los diversos modelos para el estudio de la calidad de vida laboral, entre los más importantes están el modelo de Nadler y Lawler, el modelo de Hackman y Oldhan y el modelo de Walton (Chiavenato, 2009), cada uno contempla factores, que tienen en común, siendo el más completo el modelo de Walton, que abarca un mayor número de factores a los 


\section{CALIDAD DE VIDA LABORAL EN LAS PYMES DE BRASIL: UN DIAGNÓSTICO}

que debe atenderse para un buen nivel de calidad de vida laboral, ya que abarca dimensiones

psicosociales no consideradas por los otros dos como el equilibrio entre la vida personal y laboral, la integración social y el orgullo por el trabajo: como salario, reglas y disciplina, condiciones de trabajo, integración social, equilibrio entre trabajo y vida personal, orgullo por el trabajo, facilidad para desarrollar los conocimientos y habilidades, así como que la empresa brinde al trabajador oportunidades de crecimiento y estabilidad laboral. Asimismo, Nafei (2015) propone un modelo Organizational Citizenship Behavior (OCB) para medir la calidad de vida del empleado basado en Luthans, Avey, Avolio, Norman \& Combs (2006); Podsakoff, MacKenzie, Moorman, y Richard (1990) que define a la calidad de vida laboral como la condición favorable y el entorno de beneficio de los empleados, las actitudes de bienestar y gestión de los empleados hacia los trabajadores operativos y los empleados en general.

\section{DESCRIPCIÓN DE LA CIUDAD DE PIRACICABA}

La ciudad de Piracicaba es importante para la economía de Brasil, en los últimos 10 años, por su desarrollo industrial y agrícola. Se ubica en una de las regiones más industrializadas y productivas de todo el Estado de São Paulo en Brasil. Pasó de ser una ciudad agricultura, por estar a orillas del Río Piracicaba, actualmente, es una ciudad industrializada en el país con más de cinco mil industrias, destacándose las actividades de los sectores metalúrgico, mecánico, textil, alimenticio y combustibles con la producción de petroquímicos y de alcohol (IVACE, 2015). Casi todas las ciudades importantes de Latinoamérica, han pasado por esta misma situación en su crecimiento, de tener economías agrícolas y al pasar el tiempo, convertirse en economías industriales. Lo que ha provocado, como uno de tantos factores, problemas en la gestión del capital humano. Que si bien, esta transformación de ser agrícola a industrial ha sido en promedio de 100 años (SEBRAE, 2013), aún se siguen presentando condiciones precarias y hasta nulas en la administración de las pequeñas y mediana empresas, las pymes. Entonces, ¿Cuál es el nivel en la calidad de vida laboral de los empleados en las Mypes del sector comercio y servicios en Piracicaba, Brasil? ¿Los empleados en las MyPEs del sector comercio y servicios en Piracicaba, Brasil están completamente satisfechos con su vida laboral? ¿Las MYPES del sector comercio y servicios en Piracicaba, Brasil se preocupan por la calidad de vida laboral de sus empleados? 
CALIDAD DE VIDA LABORAL EN LAS PYMES DE BRASIL: UN DIAGNÓSTICO

\section{MATERIAL Y MÉTODO}

El objetivo del presente, es analizar el nivel de calidad de vida laboral en MyPEs de Brasil, que permita identificar puntos clave para la gestión eficiente del capital humano. Se desarrolló una investigación empírica con un enfoque cuantitativo de tipo transversal, no experimental (Kerlinger, 2002), se aplicó una encuesta a empresas MyPEs en ciudad de Piracicaba en el Estado de São Paulo, Brasil. La muestra fue por conveniencia de 14 empresas clasificadas por la SEBRAE (2013), como Micro y Pequeña Empresa MyPEs. Fueron cuatro microempresas del sector comercial y 10 pequeñas empresas del sector servicios) ${ }^{1}$. El instrumento se diseñó y adaptó con base en una investigación realizada por Nafei (2015), el modelo conceptual de la investigación está descrito por las variables: esperanza-expectativa, optimismo, resiliencia, auto-eficacia (satisfacción laboral), por las variables: ambiente de trabajo, perfil del puesto, salarios y recompensas, trabajo en equipo, supervisión, participación en las decisiones (calidad de vida laboral) y por las variables: altruismo, cortesía, activismo, conciencia y moral-civismo (calidad de vida personal).

Las empresas -sujetos de estudio- se tomó en cuenta el tamaño de la empresa según la categoría de SEBRAE es una entidad privada que promueve la competitividad y el desarrollo sostenible de los emprendimientos de micro y pequeño porte (SEBRAE, 2013), que considera la cantidad de empleados (ver tabla 2).

Tabla 2

Clasificación de las MyPEs en Brasil

\begin{tabular}{|c|c|c|c|}
\hline Ordenación Jurídica & Micro Empresa & Pequeña Empresa & Mediana Empresa \\
\hline \multicolumn{4}{|l|}{ Estatuto de MIPYMES } \\
\hline Facturación bruta actual & $\mathrm{R} \$ 433.000,00$ & $\mathrm{R} \$ 2.133 .000,00$ & $\ldots \ldots \ldots \ldots$ \\
\hline \multicolumn{4}{|l|}{ SIMPLES } \\
\hline Facturación bruta anual & $\mathrm{R} \$ 240.000,00$ & $\mathrm{R} \$ 2.400 .000,00$ & $\ldots \ldots \ldots \ldots$ \\
\hline \multicolumn{4}{|l|}{ MTE/RAIS } \\
\hline $\mathrm{N}^{\circ}$ de empleados & $0-19$ & $20-99$ & $100-499$ \\
\hline
\end{tabular}

\footnotetext{
1 Las MyPEs del sector comercial eran tiendas de conveniencia, abarroteras, farmacias, papelerías, tlapalerías, ferreterías, misceláneas y boutiques de ropa, y las MyPEs del sector de servicios eran tintorerías, lavanderías, agencias publicitarias, talleres mecánicos, asesoría educativa, escuelas de educación básica, reparadoras de calzado, cocinas económicas, restaurantes, reparadoras de celulares y centros de servicio, mantenimiento y reparación de equipos de cómputo y electrodomésticos.
} 
CALIDAD DE VIDA LABORAL EN LAS PYMES DE BRASIL: UN DIAGNÓSTICO

\begin{tabular}{|c|c|c|c|}
\hline \multicolumn{4}{|l|}{ SEBRAE } \\
\hline Industria $\mathrm{N}^{*}$ de empleados & $0-19$ & $20-99$ & $100-499$ \\
\hline \multicolumn{4}{|l|}{ SEBRAE } \\
\hline Comercio y Servicios & $0-9$ & $20-99$ & $50-99$ \\
\hline $\mathrm{N}^{\circ}$ de empleados & & & \\
\hline
\end{tabular}

Fuente: (SEBRAE, 2013).

\section{RESULTADOS}

Para la presente investigación, se presentan de forma global (promedio) la estadística aplicada a las empresas comerciales y de servicios, debido a que los resultados individuales por sector variaron en menos del 5\%, entre las medias y la desviación estándar de cada sector.

La fiabilidad de los cuatro constructos y en general fue adecuada:

- Coeficiente de .853 General.

- Satisfacción del Empleado .920.

- Calidad de Vida Laboral .750.

- Calidad de Vida Personal .890.

Respecto al análisis descriptivo, se tiene que en el constructo Satisfacción del Capital Humano, en esperanza-expectativas la media es de 3.80 y la desviación estándar de 0.984; optimismo con una media de 3.50 y desviación estándar de 1.09; resiliencia con una media de 3.0 y una desviación estándar de 1.32 y para auto-eficacia se tuvo una media de 3.90 y una desviación estándar de 0.980. Como se puede observar, los empleados de las MyPEs de Brasil, no están completamente satisfechos, en su vida en términos generales. Aunque, con base en la media más alta, se considera eficaces en términos de sus actividades personales como laborales, puede decirse que poseen seguridad y autoestima al realizar lo que hacen.

Para el constructo calidad de vida laboral, los empleados de las MyPEs de Brasil, consideran que el ambiente laboral es adecuado, ya que se tiene una media de 4.00 y una desviación estándar de 0.730; perfil del puesto tiene una media de 4.40 y salarios-recompensas una media de 4.15 (desviación estándar de 0.780 y 0.730 respectivamente), lo cual se puede interpretar que los empleados están de acuerdo que su perfil del puesto tiene relación con sus capacidades (auto-eficacia) y las recompensas o salarios que perciben. Para las variables trabajo en equipo, supervisión y participación en las decisiones, parece pasar lo contrario 
CALIDAD DE VIDA LABORAL EN LAS PYMES DE BRASIL: UN DIAGNÓSTICO

(medias de 3.40, 3.59 y 3.0, respectivamente), ya que con base en los datos analizados, parece ser que no están de acuerdo en tener relaciones adecuadas con sus compañeros y sus jefes inmediatos, menos que sean tomados en cuenta en las decisiones de constructos departamento, sección de trabajo o empresa.

Respecto al constructo calidad de vida personal, los resultados no se perciben adecuados ni positivos. Desde la perspectiva de confianza y seguridad de las respuestas de los empleados a estos cuestionamientos, se tiene que estos aceptan no poseer características de bienestar personal consigo mismo, ya que en altruismo, conciencia y civismo consideran que no están de acuerdo en llevarlos del todo a cabo en su vida diario (medias de 2.8, 3.2 y 2.0, respectivamente). En cuanto a cortesía y activismo, no está tan diferente, las medias son de 3.4 y 3.3 , respectivamente.

\section{CONCLUSIONES}

Al ser la calidad de vida laboral un constructo tan complejo, multidimensional y relacionada con factores de tipo económico, psicológico y social, tanto a nivel del individuo como de la organización, como observamos en los resultados presentados, se puede concluir que la satisfacción del empleado en las empresas, están relacionadas estrecha y fuertemente con la calidad de vida laboral y personal. Un empleado satisfecho personalmente, rendirá más en la empresa, será más productivo y mayor lealtad también. Asimismo, esto influye en el nivel de vida laboral que tengan los empleados; es decir, un empleado con altos niveles de calidad de vida en su familia, en el mismo y con la empresa o puesto que tenga, repercutirá en la satisfacción integral en la empresa.

\section{REFERENCIAS BIBLIOGRÁFICAS}

Chiavenato, I. (2011). Administración de recursos humanos. El capital humano de las organizaciones (9a. ed.). México: McGraw-Hill.

García, G. y Larios, E. (2018). El clima laboral en universidades privadas con enfoque religioso católico y evangélico en Puebla-México. Revista Ide@s Concyteg. 2018(175). 
CALIDAD DE VIDA LABORAL EN LAS PYMES DE BRASIL: UN DIAGNÓSTICO

Instituto Valenciano de Competitividad Empresarial (IVACE). (2015). Oficina Económica y Comercial de España en Brasilia.

Kerlinger. (2002). Investigación del Comportamiento. México: McGraw Hill.

Luthans, F., Avey, J., Avolio, B., Norman, S. y Combs, G. (2006). Psychological capital development: Toward a micro-intervention. Journal of Organizational Behavior, 27, 387-393. Recuperado de http://dx.doi.org/10.1002/job.373

Martínez, M., Sánchez, L., Santero, R. y Marcos, M. (2009). Factores de competitividad de la Pyme española 2008. España: Fundación EOI.

Nafei, W. (2015). Meta-analysis of the impact of psychological capital on quality of work life and organizational citizenship behavior: A study on Sadat City University. International Journal of Business Administration, 6(2), 42.

Podsakoff, M., MacKenzie, S., Moorman, R. y Richard, F. (1990). Transformational Leader Behaviors and Their Effects on Followers' Trust in Leader, Satisfaction, and Organizational Citizenship Behaviors. Leadership Quarterly, 1(2), 107-142.

Saavedra, M., Milla, S. y Tapia B. (2013). Determinación de la competitividad de la PYME en el nivel micro: El caso del Distrito Federal, México: McGraw Hill.

SEBRAE -Serviço Brasileiro de Apoio às Micro e Pequenas Empresas (2013). Pequeños Negócios. Desafios e Perspectivas Sebrae Vol. 5. Recuperado de http://www.bibliotecas.sebrae.com.br/chronus/ARQUIVOS_CHRONUS/bds/bds.nsf/b449b8c1b9 3575d03b773ffadfc757bc/\$File/4508.pdf

Zamora, N. (2005). El Liderazgo en el Clima Organizacional y sus Repercusiones en la Creación de Valor (Tesis de maestría). Instituto Politécnico Nacional, México. Recuperado de http://hdl.handle.net/123456789/1135 